\title{
Controlling stray electric fields on an atom chip for experiments on Rydberg atoms
}

\author{
D. Davtyan, S. Machluf, M. L. Soudijn, J. B. Naber, N. J. van Druten, H. B. van Linden van den Heuvell, and R. J. C. Spreeuw \\ Van der Waals-Zeeman Institute, University of Amsterdam, Science Park 904, 1098 XH Amsterdam, Netherlands
}

(Received 17 October 2017; published 22 February 2018)

\begin{abstract}
Experiments handling Rydberg atoms near surfaces must necessarily deal with the high sensitivity of Rydberg atoms to (stray) electric fields that typically emanate from adsorbates on the surface. We demonstrate a method to modify and reduce the stray electric field by changing the adsorbate distribution. We use one of the Rydberg excitation lasers to locally affect the adsorbed dipole distribution. By adjusting the averaged exposure time we change the strength (with the minimal value less than $0.2 \mathrm{~V} / \mathrm{cm}$ at $78 \mu \mathrm{m}$ from the chip) and even the sign of the perpendicular field component. This technique is a useful tool for experiments handling Rydberg atoms near surfaces, including atom chips.
\end{abstract}

DOI: 10.1103/PhysRevA.97.023418

\section{INTRODUCTION}

Due to their extreme properties, atoms in a Rydberg state are interesting objects for creating strongly interacting quantum systems [1-4]. For example, Rydberg atoms strongly interact over large interatomic distances [5-8] with a van der Waals interaction that scales with principle quantum number $n$ as proportional to $n^{11}$ [9]. This interaction can be switched on and off by exciting and deexciting the atoms to and from the Rydberg state. In addition, Rydberg states have long lifetimes, proportional to $n^{3}$, required for quantum information purposes. However, the large electron orbit also leads to a large polarizability that scales as proportional to $n^{7}$ and makes Rydberg atoms sensitive to electric fields. Managing electric fields is therefore an important issue for experiments handling Rydberg atoms near the surface of an atom chip [10-12].

An attractive way to realize a scalable Rydberg quantum platform is to trap small atomic clouds in arrays of magnetic microtraps close enough to each other such that they can interact [13], using an atom chip [14]. However, a small intertrap separation $(\sim 5-10 \mu \mathrm{m})$ also implies a similarly small distance to the surface. At such a short range the stray electric fields can be prohibitively large. In fact, Rydberg atoms are used to measure electric fields $[15,16]$.

In this paper we investigate a method to control the electric field by locally affecting the surface of the atom chip with a blue laser. The mechanism behind this change is likely a combination of thermally activated desorption and light-induced atomic desorption (LIAD) [17]. Both these effects are known and used in the cold-atom community. Changing the temperature of the surface is used to decrease the stray electric field [18,19] and thus facilitate Rydberg excitation [16]. Light-induced atomic desorption has been used by many groups mostly as

Published by the American Physical Society under the terms of the Creative Commons Attribution 4.0 International license. Further distribution of this work must maintain attribution to the author(s) and the published article's title, journal citation, and DOI. a controllable source of atoms and to increase the number of atoms collected in a magneto-optical trap (MOT) [20,21].

In this work we focus our Rydberg excitation lasers onto the surface. This changes the adsorbate distribution on the surface depending on the duty cycle of one of the lasers. We probe the electric field at various distances from the chip using two-photon Rydberg spectroscopy. The measurements show that this technique can affect not only the strength of the field but also its direction. We model the adatom distribution and the resulting stray field, based on the deposition and desorption of adatom patches during the experimental cycle. We also take into account the calculated temperature profile caused by the heating laser. This technique holds promise for spatially controlling electric fields in Rydberg experiments, which is necessary for building a scalable platform for quantum information with long coherence times.

\section{EXPERIMENTAL APPARATUS AND MEASUREMENT TECHNIQUE}

Our setup for Rydberg experiments on an atom chip has been described earlier [12]. Briefly, we transfer atoms from a magneto-optical trap into a $z$-wire magnetic trap, yielding a cigar-shaped cloud of ${ }^{87} \mathrm{Rb}$ atoms with peak density of approximately $0.14 \times 10^{12} \mathrm{~cm}^{-3}$ and a temperature of $\sim 3 \mu \mathrm{K}$. We excite atoms into the $25 S_{1 / 2}$ state using a twophoton transition. The ground state $\left|5 S_{1 / 2}, F=2, m_{F}=2\right\rangle$ is coupled to the Rydberg state through the intermediate state $\left|5 P_{3 / 2}, F^{\prime}=3\right\rangle$ with a large blue intermediate-state detuning $\Delta=2 \pi \times 1.5 \mathrm{GHz}$ to prevent population of the intermediate state. In Fig. 1(a) we show the level scheme of the two-photon Rydberg excitation using two lasers: a red laser $(780 \mathrm{~nm})$ with a power of $P_{\mathrm{r}}=35 \mu \mathrm{W}$ and a blue laser ( $\left.\sim 480 \mathrm{~nm}\right)$ with a power of $P_{b}=55 \mathrm{~mW}$. Both laser beams have waists $\left(1 / e^{2}\right.$ radius $)$ of $w_{0} \approx 100 \mu \mathrm{m}$. This gives a two-photon Rabi frequency $\Omega=$ $\frac{\Omega_{\mathrm{r}} \Omega_{\mathrm{b}}}{2 \Delta} \approx 2 \pi \times 276 \mathrm{kHz}$. The one-photon Rabi frequencies $\left(\Omega_{\mathrm{r}}\right.$ and $\Omega_{\mathrm{b}}$ ) were calculated using the Alkali Rydberg Calculator software [22]. For the measurements discussed here the bottom field of the magnetic trap $B_{\mathrm{IP}}$ is between 1 and $3 \mathrm{G}$, depending on the distance from the surface of the chip. The Rydberg transition should not be affected by the magnetic field because 
(a)

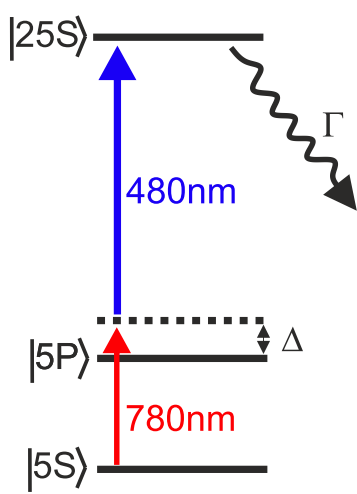

(c)

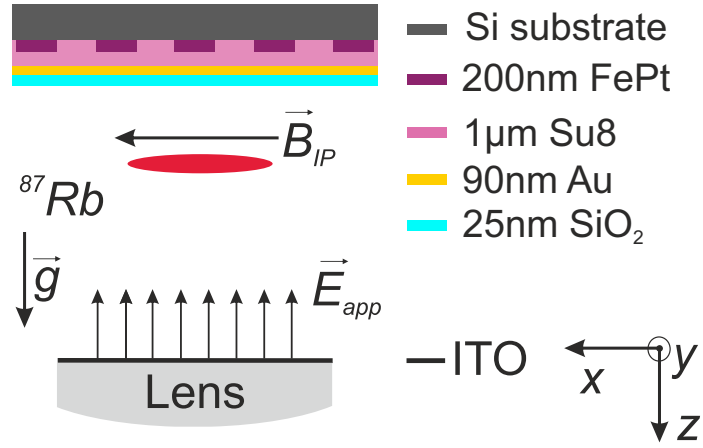

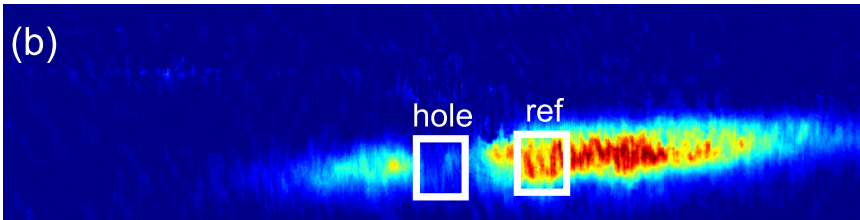

$100 \mu \mathrm{m}$
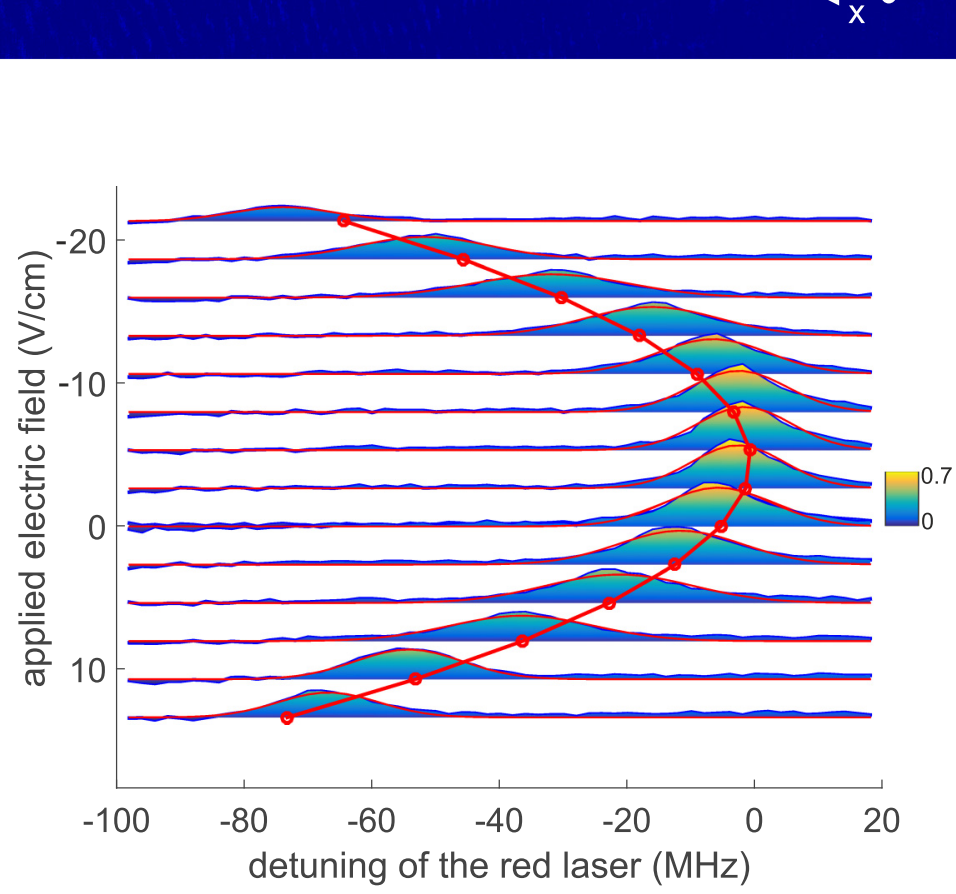

FIG. 1. (a) Two-photon excitation scheme to the Rydberg state. (b) Example of an absorption image of an atomic cloud, trapped in a magnetic $z$-wire trap. The normalized depletion is obtained by taking the ratio of the number of atoms in the "hole" area (where the excitation lasers are focused) and the number of atoms in the "ref" area. (c) Sketch of the setup (not to scale). The high-numerical-aperture lens is coated with ITO, which allows the application of a voltage between the lens and the chip to compensate for the $z$ component of the stray electric field. In order to probe the electric field on different heights, the atomic cloud (red) can be moved up and down by varying the current through the trapping wire ( $z$ wire, not shown). The chip contains a stack of layers of different materials, including a 1- $\mu$ m SU8 polymer layer which provides thermal insulation and leads to heating of the surface (see Sec. IV B). (d) Example of a Stark map at a height of $163 \mu \mathrm{m}$. For each value of the voltage between the chip and the lens, a spectrum is taken by scanning the detuning of the red laser and measuring the normalized depletion. The red line is a fit to the Stark map; the values of $E_{z}$ and $E_{x, y}$ are retrieved from the fit.

our light polarization allows only for excitation of the $m_{j}=$ $1 / 2$ state, which experiences the same Zeeman shift as the $|2,2\rangle$ ground state.

In Fig. 1(b) we show an example of an absorption image of an atomic cloud in the $z$-wire trap. When excited into a Rydberg state, atoms have a high probability either to decay into an untrappable state or to be ionized and escape from the trap. Thus we observe a decrease in the number of trapped atoms in the area where the two laser beams are focused. The exposure time of the blue excitation laser was varied between $35 \mathrm{~ms}$ and $18 \mathrm{~s}$ and the exposure time of the red excitation laser was kept fixed at $100 \mu$ s for all the measurements. Imaging of the cloud was done in trap $100 \mu$ s after the depletion pulse, so atoms from the neighboring areas do not have time to refill the depleted area.

In Fig. 1(c) we sketch the local environment of the atomic cloud. The in-vacuum lens $(f=18.75 \mathrm{~mm}$ and numerical aperture equal to 0.4 ), through which the Rydberg and absorption imaging lasers are focused, is coated with a conductive material, indium tin oxide (ITO), so that an electric field $E_{\text {app }}$ between the lens and the chip can be applied and the $z$ component of the stray electric field $E_{\mathrm{z}}$ can be compensated. By focusing the two lasers we excite part of the atomic cloud and observe a depletion because of the Rydberg atoms escaping the trap. We then scan the frequency of the red laser and record the loss of atoms. We take spectra for different voltages applied between the chip and the lens. This results in Stark maps such as that shown in Fig. 1(d). The observed peaks are symmetrically broadened; this can be partially a result of resonant dipole-dipole interaction between the excited $25 S$ state and the neighboring $P$ states, to which the atoms can decay through blackbody radiation [8,23,24]. Following [23], we estimate our linewidth broadening to be approximately $2 \pi \times 3 \mathrm{MHz}$, while the minimum measured linewidth is approximately $2 \pi \times 6 \mathrm{MHz}$; this is in agreement with the approximate theory. Closer to the surface of the chip, the spectral widths increase. This could be partly due to an increase of density as the cloud is compressed closer 


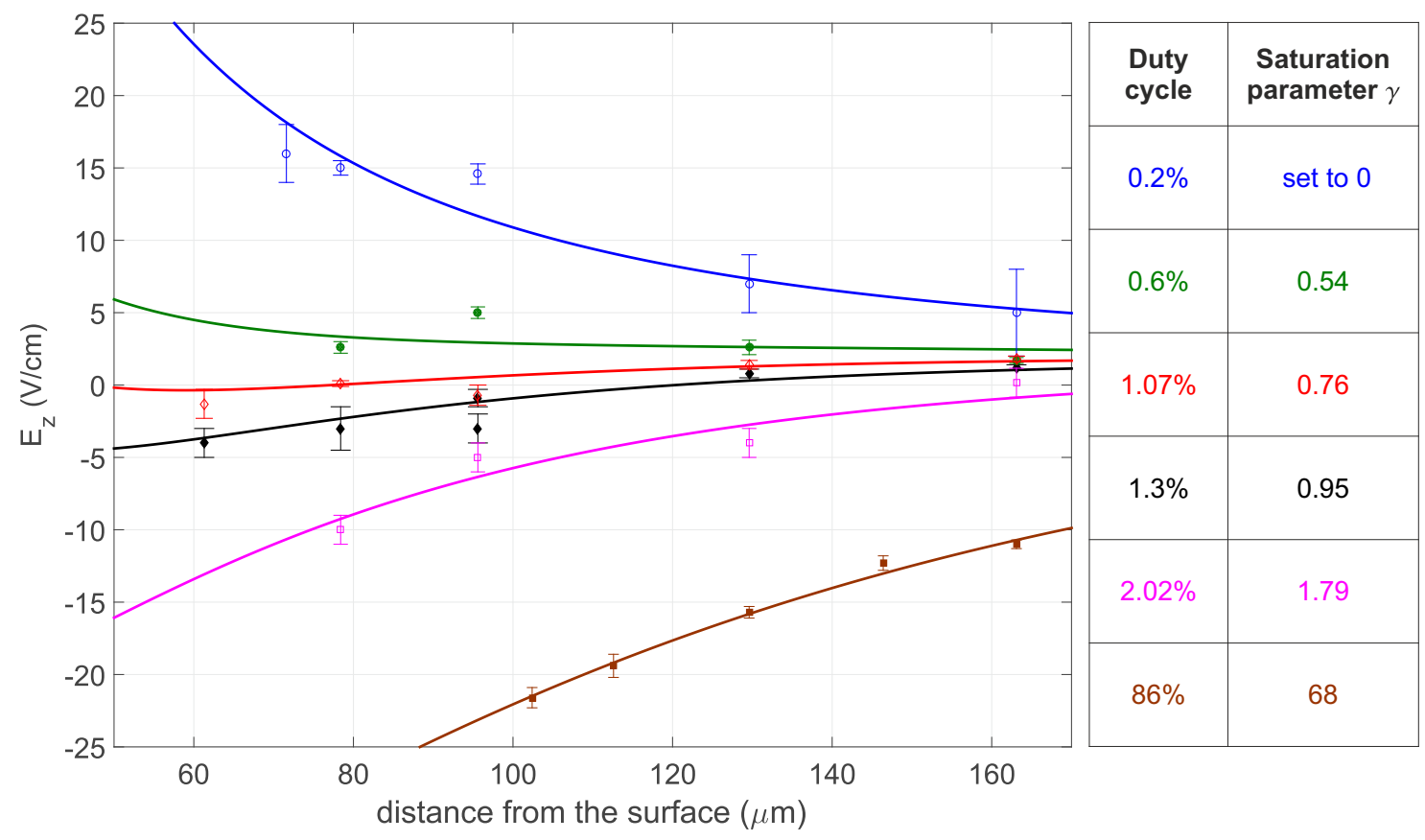

FIG. 2. Dependence of the electric field on the distance from the chip for different duty cycles of the blue laser. The result of the measurement of $E_{z}$ is shown with error bars. The solid lines show the result of a single collective fit (see the text for details).

to the surface. Another possible contribution to the linewidth broadening could be increased electric-field gradients.

The Stark map has a parabolic shape, as expected for a Ryberg $S$ state for small fields. In the center of the parabola the $z$ component of the stray electric field is compensated by the applied field. Thus the applied compensation field yields a measurement of the stray field $z$ component. The zero-frequency detuning corresponds to the resonance, as calibrated in a spectroscopy vapor cell at room temperature in a field-free environment using electromagnetically induced transparency [25,26]. Thus the remaining frequency shift of the vertex of the parabola is due to the uncompensated $E_{x, y}$ (parallel to the chip surface) component of the stray electric field. Rydberg energy levels are calculated by diagonalizing the Stark Hamiltonian using $E_{\text {app }}$ as an input parameter and using $E_{x, y}$ and $E_{z}$ as fitting parameters. To obtain a good fit, we also introduce as an extra fitting parameter a factor that enhances $E_{\text {app }}-E_{z}$. This is necessary because some of the measured Stark maps have different (typically larger) curvature than what is expected from the polarizability of the investigated Rydberg state. In some measurements we also observe an asymmetry between the two branches of the parabola which can also increase the apparent parabola curvature, in a few cases up to a factor of 4 . By fitting separately the two branches of the parabola, we verified numerically that this asymmetry does not affect the position of the vertex to within the error bars. We speculate that these effects may be due to spatially dependent deposition of ions or electrons, originating from ionized Rydberg atoms. On the surface these charges form dipoles with their mirror charge in the gold layer and contribute to the stray field. A full understanding of this complex interplay is beyond the scope of this paper. Finally, by changing the current in the $z$ wire we vary the distance between the atomic cloud and the atom chip and retrieve the dependence of the electric field $E_{z}$ on the distance to the chip.

\section{EXPERIMENTAL RESULTS}

In previous experiments we observed large stray electric fields above a gold surface [27] and even $\sim 10$ times larger fields above silica-coated gold [12]. However, in other studies [16] significant reduction of the stray electric field was achieved by forced desorption of the adatoms. In our experiment we use the focused blue excitation laser to induce local rubidium desorption through LIAD and heating of the chip surface. We vary the average laser power, incident on the chip, by the fractional time (duty cycle) of the blue laser during the experimental cycle. The pulse length of the red excitation laser (100 $\mu \mathrm{s})$ is kept constant for all the measurements. We then measure the $z$ component of the electric field for different duty cycles of the blue laser. Because the power and the exposure time of the red Rydberg laser and the imaging laser are much lower than for the blue one, their effect on the chip surface is negligible. The result of the measurement of $E_{z}$ on different heights for different duty cycles is shown in Fig. 2. The results are reproducible to within the error bars (1-2 V/cm): After changing the duty cycle and letting the system reach the new steady state (typically $\sim 2.5-3 \mathrm{~h}$ ) we measure $E_{z}$, corresponding to the new duty cycle. We checked that the measured electric field does not depend on the previous value of the duty cycle by comparing with different ordering of duty cycles. The observed values of the frequency shift of the parabola vertex correspond to the parallel component $[(x, y)$ plane] of the stray field between $(0 \pm 1) \mathrm{V} / \mathrm{cm}$ at $\sim 160 \mu \mathrm{m}$ and increasing to $(10 \pm 2) \mathrm{V} / \mathrm{cm}$ at $\sim 60 \mu \mathrm{m}$. No dependence of $E_{x, y}$ on the duty cycle was observed. The in-plane field can be explained by a spatial mismatch [breaking the symmetry, as can be seen from Fig. 1(b)] between the excitation beam and the Gaussian patches of the dipole distribution. The error bars are standard errors for the fits of the measured Stark maps and the solid lines show the results of the fitted electric field $E_{z}$ using the model described below. 


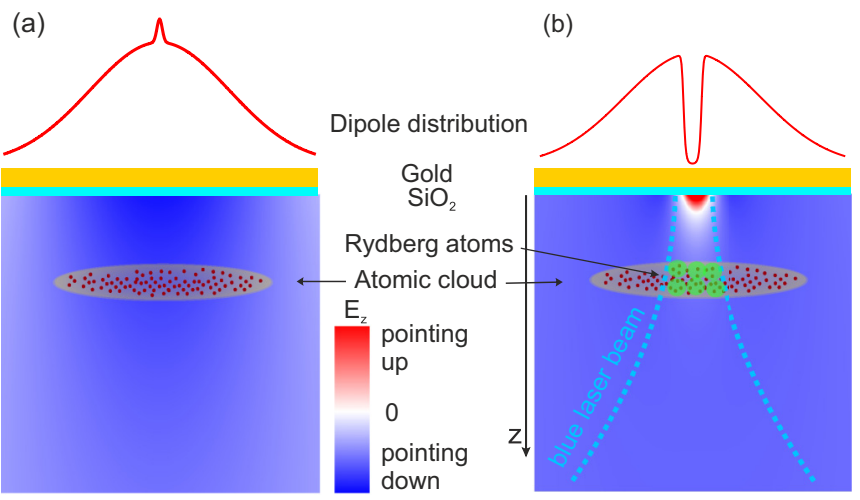

FIG. 3. Simulation of the electric field $E_{z}$ due to a distribution of dipoles on the surface. The red curves show the dipole distribution. The gold and the cyan parts show the two outermost layers of the chip, the gold and the silica, respectively. The $E_{z}$ is indicated by color, blue (red) indicating the electric field pointing down (up). (a) Short duty cycle of the blue laser (see Sec. IV A) and (b) adsorbate distribution with a Gaussian hole in it, corresponding to a long duty cycle of the blue laser. In the area where the blue laser (dashed line) hits the surface, a hole in the adsorbate layer occurs due to thermal and light-induced atomic desorption.

The experimental cycle time is $\sim 21 \mathrm{~s}$. The uppermost blue data show the determined $E_{z}$ for the shortest exposure time of the blue laser of $\sim 35 \mathrm{~ms}$ and thus $0.2 \%$ duty cycle. The electric field increases closer to the surface of the chip. However, for higher duty cycles $E_{z}$ decreases and at some point even changes sign. Below we present a model to describe this behavior.

\section{MODEL}

\section{A. Electric-field simulation}

To explain our results we use a model based on a distribution of electric dipoles, formed by $\mathrm{Rb}$ adsorbates on the silica surface. An example is shown in Fig. 3, where the chip (gold and silica layers) is shown with a dipole distribution (red curve). The positive direction of the $z$ axis coincides with gravity. The value of $E_{z}$ is indicated by color intensity. Blue (red) indicates $E_{z}$ pointing down (up). White color means the absence of $E_{z}$. First we assume that the distribution [see Fig. 3(a)] is a double Gaussian, so the electric field points down everywhere. The narrow Gaussian corresponds to atoms released from the $z$-wire trap. The wide Gaussian distribution represents atoms released from the MOT. Once the blue laser is applied to the center of the dipole distribution for a long enough time, it causes local desorption of the dipoles. The resulting distribution is shown in Fig. 3(b). It looks like the original distribution with a hole in the center. In the new case the field lines change direction from down to up in the center of the hole and a small region with no stray field appears (the white area).

Experimentally, after the blue laser starts to act on the surface, the desorption of adatoms is locally enhanced. We attribute this effect to a combination of LIAD and a local increase of the temperature. The temperature of the spot where the beam hits the surface increases instantly (on the experimental time scale) and accelerates the desorption of the adatoms. When the blue laser pulse ends, the temperature of the heated area instantly cools down to room temperature. The density of the remaining adatoms (and thus the dipole distribution) depends on the exposure time of the blue laser during the experimental cycle (duty cycle).

The dependence of the electric field on the dipole distribution was simulated using MATLAB software. The $z$ component of the electric field at a distance $z$ from the chip produced by a single dipole on the surface is

$$
E_{z}^{\mathrm{s}}(0,0, z)=\frac{P}{4 \pi \epsilon_{0}} \frac{1}{\left(x^{2}+y^{2}+z^{2}\right)^{3 / 2}}\left[3 \frac{z^{2}}{x^{2}+y^{2}+z^{2}}-1\right],
$$

where $(x, y, 0)$ are the coordinates of the dipole and $P$ is the dipole moment of a single $\mathrm{Rb}$ adatom. For $P$ we take the value $P=12$ D $[12,16]$ multiplied by a correction factor $\frac{\epsilon+1}{\epsilon} \approx 1.25$ ( $\epsilon=3.9$ is the dielectric constant of silica) to account for image dipoles due to the gold surface $[18,19,28,29]$. The $z$ axis coincides with the Rydberg excitation beams. The electric field $E_{z}$ at the point $(0,0, z)$ is then calculated as

$$
E_{z}(0,0, z)=\iint_{S_{\mathrm{ch}}} E_{z}^{\mathrm{s}}(0,0, z) \rho_{\mathrm{d}}(x, y) d x d y,
$$

where $\rho_{\mathrm{d}}$ is the surface dipole density. Equation (2) is integrated over the area of the chip $S_{\mathrm{ch}}=L_{x} L_{y}$, with $L_{x}=16 \mathrm{~mm}$ and $L_{y}=20 \mathrm{~mm}$.

We simulate the dipole distribution as two Gaussian patches: The first one is narrow with the size of the atomic cloud in the $z$-wire trap $\left(\sigma_{\mathrm{n}, \mathrm{x}} \approx 170 \mu \mathrm{m}\right.$ and $\left.\sigma_{\mathrm{n}, \mathrm{y}} \approx 21 \mu \mathrm{m}\right)$ and the second one is wide due to atoms expanding from the MOT $\left(\sigma_{\mathrm{w}}=4.5 \mathrm{~mm}\right)$. The size of the hole in the dipole distribution $\left(w_{\mathrm{h}}=2 \times \sigma_{\mathrm{h}}=100 \mu \mathrm{m}\right)$ is taken to coincide with the waist of the blue laser beam. This choice is obvious for the LIAD mechanism. For thermal desorption our model (see Sec. IV B) indicates that the temperature profile is only slightly wider than $w_{\mathrm{h}}$. We find that for obtaining good fits at large duty cycles it is necessary to assume that the hole size broadens. We describe this by introducing a saturation mechanism. This saturation can be justified by the fact that the measured electric field is very sensitive to the duty cycle for small duty cycles, but loses its sensitivity for larger duty cycles. Also, the fact that the data for the case of the largest duty cycle are the least noisy suggests that the surface is then clean and the measurement suffers less from shot to shot fluctuations of the adsorbant number.

We describe the total dipole density as

$$
\rho_{\mathrm{d}}=\left(\rho_{\mathrm{w}}+\rho_{\mathrm{n}}\right)(1-h),
$$

where $h$ is the hole profile, $0<h<1$, with $h \rightarrow 1$ corresponding to full depletion. In Eq. (3) $\rho_{\mathrm{w}}$ is the broad truncated Gaussian distribution

$$
\rho_{\mathrm{w}}(x, y)= \begin{cases}\frac{N_{\mathrm{w}}}{2 \pi \sigma_{\mathrm{w}}^{2}} e^{-\left(x^{2}+y^{2}\right) / 2 \sigma_{\mathrm{w}}^{2}} & \text { for }|x, y|<L_{x, y} / 2 \\ 0 & \text { otherwise, }\end{cases}
$$

with $N_{\mathrm{w}}$ the number of atoms in the Gaussian distribution. The narrow Gaussian part is

$$
\rho_{\mathrm{n}}(x, y)=\frac{N_{\mathrm{n}}}{2 \pi \sigma_{\mathrm{n}, \mathrm{x}} \sigma_{\mathrm{n}, \mathrm{y}}} e^{-x^{2} / 2 \sigma_{\mathrm{n}, \mathrm{x}}^{2}-y^{2} / 2 \sigma_{\mathrm{n}, \mathrm{y}}^{2}},
$$


with $N_{\mathrm{n}}$ the number of atoms in the narrow distribution. The hole profile is modeled as

$$
h(x, y)=\frac{\gamma e^{-\left(x^{2}+y^{2}\right) / 2 \sigma_{\mathrm{h}}^{2}}}{1+\gamma e^{-\left(x^{2}+y^{2}\right) / 2 \sigma_{\mathrm{h}}^{2}}} .
$$

We introduce here a heuristic saturation parameter $\gamma$ used as a fitting parameter in the model, as are $N_{\mathrm{w}}$ and $N_{\mathrm{n}}$. This saturation model for the depletion provides the necessary broadening mechanism for the dipole distribution.

For the sake of stability of the fit, we set $\gamma=0$ for the $0.2 \%$ duty cycle (blue open circles in Fig. 2). This is justified by the fact that this is the shortest duty cycle which still allows us to see the Rydberg depletion in the atomic cloud and is the best approximation to the dipole distribution without the effect of the blue laser. Also for stability reasons $N_{\mathrm{n}}$ is set to 0 for the highest duty cycle data ( $86 \%$ duty cycle), justified by the fact that for such a large saturation the small dipole distribution effectively vanishes. All the curves are simultaneously fitted to a single set of parameters: $N_{\mathrm{w}}$ is the same for all six data sets, $N_{\mathrm{n}}$ is the same for all duty cycles except the highest $86 \%$, and $\gamma$ is individually fitted for each data set. The result of the fit is shown in Fig. 2. The error bars on the data are based on the fit to the Stark maps. The curves are the result of the fit of the hole in the dipole distribution. In the table on the right-hand side of Fig. 2, for each curve the corresponding values of the duty cycle and saturation parameter are shown.

The fit gives the values for the dipole distribution: $N_{\mathrm{w}}=(6.47 \pm 0.02) \times 10^{13}$ dipoles and $N_{\mathrm{n}}=(4.13 \pm$ $0.01) \times 10^{9}$ dipoles. This gives the peak surface dipole density $\rho_{\max }=7.03 \times 10^{5}$ atoms $/ \mu \mathrm{m}^{2}$. The found dipole density gives a minimum average adatom spacing of $\sim 1.2 \mathrm{~nm}$. This is of the same order as in other studies: In [16] the estimated surface density and average interadatom spacing are $4 \times 10^{5}$ atoms $/ \mu \mathrm{m}^{2}$ and $\sim 1.5 \mathrm{~nm}$, respectively. The $x$ and $y$ cuts of the dipole distributions for different values of $\gamma$ are shown in Fig. 4. For the largest value of $\gamma=68$ (brown curve) the saturation is clearly visible as a widening of the hole.

\section{B. Temperature simulation}

In order to understand the heating mechanism of the chip during the experiment, the temperature of the chip was simulated. As shown in Fig. 1(c), the atom chip is a stack of layers with different materials. The adatoms are adsorbed and desorbed from silica $(\sim 25 \mathrm{~nm}$ layer). There is a thin layer of gold $(90 \mathrm{~nm})$ for the mirror MOT and a $1-\mu \mathrm{m}$ layer of dielectric (SU8) for planarization of the magnetic structure $(200 \mathrm{~nm} \mathrm{FePt})$, which is used to create magnetic microtraps. The last layer of the chip is a relatively thick $(\sim 300 \mu \mathrm{m})$ silicon substrate. The chip is clipped to a metal construction, which is a good heat conductor. We assume the connection of the chip to the metal construction to be at room temperature $296 \mathrm{~K}$. The laser heats the chip surface locally. The laser beam has a Gaussian profile with a waist of $\sim 100 \mu \mathrm{m}$ and power of $55 \mathrm{~mW}$. We estimate the absorption of the blue laser power by the chip to be between $70 \%$ and $80 \%$. The heating problem can be solved semianalytically. We find that the center of the laser beam heats up the chip surface by $\sim 11-13 \mathrm{~K}$ above room temperature, which leads to the enhanced desorption of the $\mathrm{Rb}$ atoms. The temperature distribution closely follows the
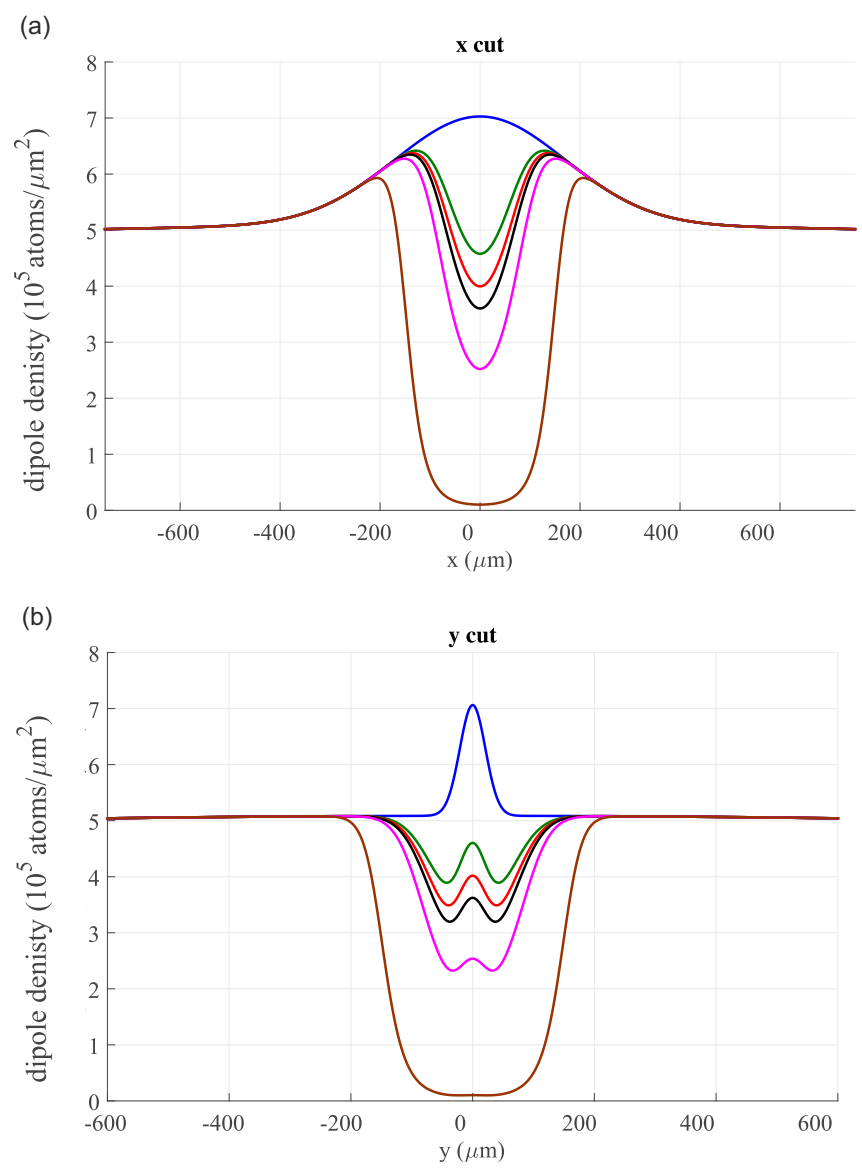

FIG. 4. Plot of (a) the $x$ cut and (b) the $y$ cut of dipole distributions from the data fits for different duty cycles (see Fig. 2). The distribution consists of a two-dimensional Gaussian on a broad layer of dipoles and a saturated hole in it.

Gaussian form of the laser beam with a root-mean-square width $\sigma_{T} \approx 53 \mu \mathrm{m}$, only slightly larger than the laser beam $\sigma_{\text {laser }}=$ $50 \mu \mathrm{m}$. The temperature profile resembles the laser beam profile almost perfectly because the characteristic length scale of the temperature change $r_{0}=\sqrt{D d \lambda_{\mathrm{Au}} / \lambda_{\mathrm{SU} 8}} \approx 12 \mu \mathrm{m}$ is much smaller than the beam diameter, where $D$ and $d$ are the thicknesses of the gold and SU8 layers, respectively, and $\lambda_{\mathrm{Au}}=318 \mathrm{~W} / \mathrm{m} \mathrm{K}$ and $\lambda_{\text {SU8 }}=0.2 \mathrm{~W} / \mathrm{m} \mathrm{K}$ are the thermal conductivities. The effective heating of the surface is due to the fact that gold is a relatively bad mirror for the blue light, but mostly because SU8 is a very good thermal insulator compared to gold.

\section{DISCUSSION}

By varying the average time of the blue laser acting on the chip we are able to control the $z$ component of the stray electric field above the chip surface. We attribute the effect dependence of $E_{z}$ on the duty cycle to a combination of thermal desorption and LIAD [20]. Usually, for LIAD UV or violet light is used [30]; however, in some special cases of low adsorption energy the LIAD effect is seen even in the red wavelength range [17]. In our case, even though the blue photon energy is not particularly high, the laser intensity is several orders of magnitudes higher than in usual LIAD 
experiments. Estimates for thermal desorption and LIAD based on the numbers provided in $[16,30]$ suggest that the desorption is dominated by LIAD due to the high beam intensity, while the mild local heating only contributes in a minor way. As we estimate the $\mathrm{Rb}$ coverage of our surface to be only a few percent, we do not expect that the surface electronic structure is modified to an extent that photoelectrons could be released [16,31]. Both the precise adsorption-desorption mechanism in our experiment and the dynamics of this process are subjects for future research.

Despite the strong electric-field reduction in our experiment, no reliable Rydberg excitation could be created in the microtraps at an $\sim 10 \mu \mathrm{m}$ distance to the surface. As the atomic cloud approaches the surface of the chip we see broadening and suppression of the excitation spectra. This prevents Rydberg excitation closer than $\sim 40 \mu \mathrm{m}$ from the chip, whereas the microtraps are located 6-8 $\mu \mathrm{m}$ from the surface of the chip. As mentioned before, one possible broadening mechanism is resonant dipole-dipole interaction. Another source of spectral broadening and instability of the stray electric field can be the creation of free charges due to the Rydberg ionization close to the surface and the deposition of those charges onto the surface.

\section{CONCLUSION}

We have demonstrated and analyzed a technique to control stray electric fields in atom chip Rydberg experiments, changing the local adsorbate dipole distribution by changing the surface conditions with one of the two Rydberg excitation lasers. This effect of an excitation laser changing locally the surface dipole distribution through heating and/or LIAD and thus changing the conditions of the experiment therefore has to be taken into account in the design of Rydberg experiments on a chip.

The method presented in this paper provides a path forward towards stable Rydberg excitation closer to the surface of an atom chip, in particular in magnetic microtraps. The geometry of the affected area can be further optimized by changing the beam parameters, for example, by using a spatial light modulator $[32,33]$.

\section{ACKNOWLEDGMENTS}

Our work was financially supported by the Netherlands Organization for Scientific Research. We also acknowledge financial support from the EU H2020 FET Proactive project RySQ (No. 640378).
[1] M. Saffman, T. G. Walker, and K. Mølmer, Quantum information with Rydberg atoms, Rev. Mod. Phys. 82, 2313 (2010).

[2] H. Bernien, S. Schwartz, A. Keesling, H. Levine, A. Omran, H. Pichler, S. Choi, A. Zibrov, M. Endres, M. Greiner, V. Vuletic, and M. D. Lukin, Probing many-body dynamics on a 51-atom quantum simulator, Nature (London) 551, 579 (2017).

[3] M. Saffman, Quantum computing with atomic qubits and Rydberg interactions: Progress and challenges, J. Phys. B 49, 202001 (2016).

[4] R. Heidemann, U. Raitzsch, V. Bendkowsky, B. Butscher, R. Loew, L. Santos, and T. Pfau, Evidence for Coherent Collective Rydberg Excitation in the Strong Blockade Regime, Phys. Rev. Lett. 99, 163601 (2007).

[5] J. Zeiher, R. van Bijnen, P. Schauß, S. Hild, J. Choi, T. Pohl, I. Bloch, and C. Gross, Many-body interferometry of a Rydbergdressed spin lattice, Nat. Phys. 12, 1095 (2016).

[6] H. Labuhn, D. Barredo, S. Ravets, S. de Léséleuc, T. Macrì, T. Lahaye, and A. Browaeys, Tunable two-dimensional arrays of single Rydberg atoms for realizing quantum Ising models, Nature (London) 534, 667 (2016).

[7] K. Singer, J. Stanojevic, M. Weidemüller, and R. Côté, Longrange interactions between alkali Rydberg atom pairs correlated to the $n s-n s, n p-n p$ and $n d-n d$ asymptotes, J. Phys. B 38, S295 (2005).

[8] C. S. E. van Ditzhuijzen, A. F. Koenderink, J. V. Hernández, F. Robicheaux, L. D. Noordam, and H. B. van Linden van den Heuvell, Spatially Resolved Observation of Dipole-Dipole Interaction Between Rydberg Atoms, Phys. Rev. Lett. 100, 243201 (2008).

[9] T. F. Gallagher, Rydberg Atoms (Cambridge University Press, Cambridge, 1994).

[10] C. Hermann-Avigliano, R. Celistrino Teixeira, T. L. Nguyen, T. Cantat-Moltrecht, G. Nogues, I. Dotsenko, S. Gleyzes, J. M.
Raimond, S. Haroche, and M. Brune, Long coherence times for Rydberg qubits on a superconducting atom chip, Phys. Rev. A 90, 040502(R) (2014).

[11] N. Cisternas, J. de Hond, G. Lochead, R. J. C. Spreeuw, H. B. van Linden van den Heuvell, and N. J. van Druten, Characterizing the local vectorial electric field near an atom chip using Rydbergstate spectroscopy, Phys. Rev. A 96, 013425 (2017).

[12] J. Naber, S. Machluf, L. Torralbo-Campo, M. L. Soudijn, N. J. van Druten, H. B. van Linden van den Heuvell, and R. J. C. Spreeuw, Adsorbate dynamics on a silica-coated gold surface measured by Rydberg Stark spectroscopy, J. Phys. B 49, 094005 (2015).

[13] V. Y. F. Leung, D. R. M. Pijn, H. Schlatter, A. Torralbo-Campo, A. L. La Rooij, G. B. Mulder, J. B. Naber, M. L. Soudijn, A. Tauschinsky, C. Abarbanel, B. Hadad, E. Golan, R. Folman, and R. J. C. Spreeuw, Magnetic-film atom chip with $10 \mu \mathrm{m}$ period lattices of microtraps for quantum information science with Rydberg atoms., Rev. Sci. Instrum. 85, 053102 (2014).

[14] V. Y. F. Leung, A. Tauschinsky, N. J. van Druten, and R. J. C. Spreeuw, Microtrap arrays on magnetic film atom chips for quantum information science, Quantum Inf. Process. 10, 955 (2011).

[15] R. P. Abel, C. Carr, U. Krohn, and C. S. Adams, Electrometry near a dielectric surface using Rydberg electromagnetically induced transparency, Phys. Rev. A 84, 023408 (2011).

[16] J. A. Sedlacek, E. Kim, S. T. Rittenhouse, P. F. Weck, H. R. Sadeghpour, and J. P. Shaffer, Electric Field Cancellation on Quartz by $\mathrm{Rb}$ Adsorbate-Induced Negative Electron Affinity, Phys. Rev. Lett. 116, 133201 (2016).

[17] M. Meucci, E. Mariotti, P. Bicchi, C. Marinelli, and L. Moi, Light-induced atom desorption, Europhys. Lett. 9, 639 (1994).

[18] J. M. McGuirk, D. M. Harber, J. M. Obrecht, and E. A. Cornell, Alkali-metal adsorbate polarization on conducting and insulat- 
ing surfaces probed with Bose-Einstein condensates, Phys. Rev. A 69, 062905 (2004).

[19] J. M. Obrecht, R. J. Wild, and E. A. Cornell, Measuring electric fields from surface contaminants with neutral atoms, Phys. Rev. A 75, 062903 (2007).

[20] A. Burchianti, A. Bogi, C. Marinelli, C. Maibohm, E. Mariotti, and L. Moi, Reversible Light-Controlled Formation and Evaporation of Rubidium Clusters in Nanoporous Silica, Phys. Rev. Lett. 97, 157404 (2006).

[21] C. Klempt, T. Van Zoest, T. Henninger, O. Topic, E. Rasel, W. Ertmer, and J. Arlt, Ultraviolet light-induced atom desorption for large rubidium and potassium magneto-optical traps, Phys. Rev. A 73, 013410 (2006).

[22] N. Šibalić, J. D. Pritchard, C. S. Adams, and K. J. Weatherill, ARC: An open-source library for calculating properties of alkali Rydberg atoms, Comput. Phys. Commun. 220, 319 (2017).

[23] E. A. Goldschmidt, T. Boulier, R. C. Brown, S. B. Koller, J. T. Young, A. V. Gorshkov, S. L. Rolston, and J. V. Porto, Anomalous Broadening in Driven Dissipative Rydberg Systems, Phys. Rev. Lett. 116, 113001 (2016).

[24] K. Afrousheh, P. Bohlouli-Zanjani, D. Vagale, A. Mugford, M. Fedorov, and J. D. D. Martin, Spectroscopic Observation of Resonant Electric Dipole-Dipole Interactions Between Cold Rydberg Atoms, Phys. Rev. Lett. 93, 233001 (2004).

[25] K. Boller, A. Imamoglu, and S. E. Harris, Observation of Electromagnetically Induced Transparency, Phys. Rev. Lett. 66, 2593 (1991).
[26] A. K. Mohapatra, T. R. Jackson, and C. S. Adams, Coherent Optical Detection of Highly Excited Rydberg States Using Electromagnetically Induced Transparency, Phys. Rev. Lett. 98, 113003 (2007).

[27] A. Tauschinsky, R. M. T. Thijssen, S. Whitlock, H. B. van Linden van den Heuvell, and R. J. C. Spreeuw, Spatially resolved excitation of Rydberg atoms and surface effects on an atom chip, Phys. Rev. A 81, 063411 (2010).

[28] R. G. Barrera, O. Guzmán, and B. Balaguer, Point charge in a three-dielectric medium with planar interfaces, Am. J. Phys. 46, 1172 (1978).

[29] F. M. Pont and P. Serra, Comment on "Point charge in a threedielectric medium with planar interfaces" [Am. J. Phys. 46, 1172 (1978)], Am. J. Phys. 83, 475 (2015).

[30] L. Torralbo-Campo, G. D. Bruce, G. Smirne, and D. Cassettari, Light-induced atomic desorption in a compact system for ultracold atoms, Sci. Rep. 5, 14729 (2015).

[31] M. Brause, D. Ochs, J. Günster, T. Mayer, B. Braun, V. Puchin, W. Maus-Friedrichs, and V. Kempter, Cs adsorption on oxide films $\left(\mathrm{Al}_{2} \mathrm{O}_{3}, \mathrm{MgO}, \mathrm{SiO}_{2}\right)$, Surf. Sci. 383, 216 (1997).

[32] F. Nogrette, H. Labuhn, S. Ravets, D. Barredo, L. Béguin, A. Vernier, T. Lahaye, and A. Browaeys, Single-Atom Trapping in Holographic 2D Arrays of Microtraps with Arbitrary Geometries, Phys. Rev. X 4, 021034 (2014).

[33] J. B. Naber, J. Vos, R. J. Rengelink, R. J. Nusselder, and D. Davtyan, Optical techniques for Rydberg physics in lattice geometries, Eur. Phys. J. Spec. Top. 225, 2785 (2016). 